\title{
Advanced Network Research at Fermilab
}

Dr. Wenji Wu

ECE Department Distinguished Seminar

September 18, 2019 


\section{Agenda}

- About Fermilab

- Why do we need advanced network research at Fermilab?

- Advanced network facilities at Fermilab

- Major research projects

- WireCap: a novel packet capture engine for commodity NICs in high-speed networks

- http://wirecap.fnal.gov

- mdtmFTP: a high performance data transfer tool (funded by DOE ASCR, \$1.5M)

- http://mdtm.fnal.gov

- BigData Express (funded by DOE ASCR, \$2.2M)

- http://bigdataexpress.fnal.gov

- Quantum network research (funded by DOE ASCR, \$3.2M) 


\section{Fermilab is America's particle physics and accelerator laboratory.}

We bring the world together to solve the mysteries of matter, energy, space and time.

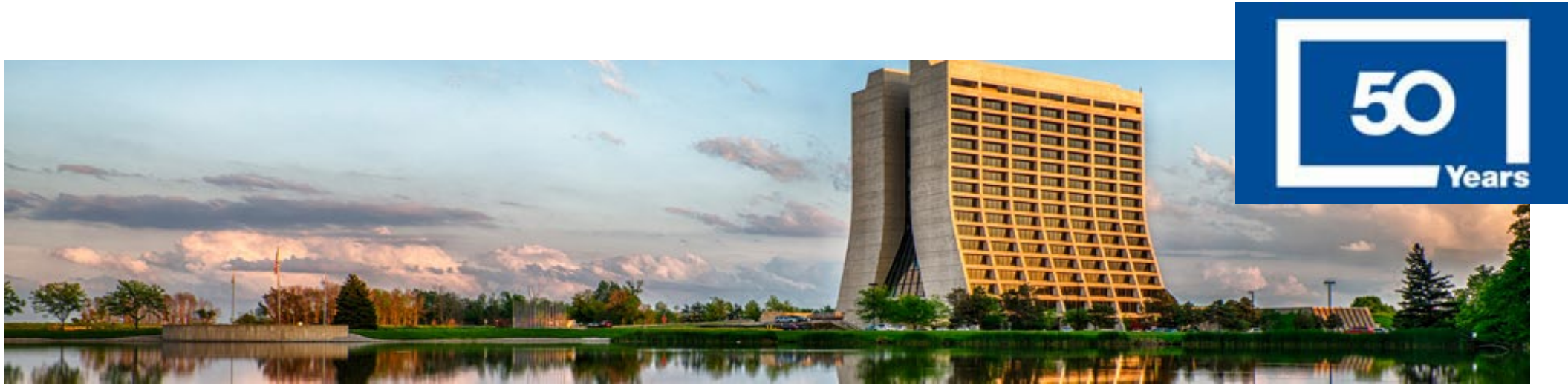

- Over 50 years at the forefront of discovery in high energy physics (top \& bottom quarks, tau neutrino,...)

- We build and operate High Power (MW), High reliability Accelerators. Funded by DOE Office of Science.

- Largest U.S. Accelerator complex, 6800 acre site, $\sim \$ 400 \mathrm{M} / \mathrm{yr}$ budget, nearly 1800 staff, > 3200 users.

- Next big project: Long Baseline Neutrino Facility and Deep Underground Neutrino Experiment 


\section{Why Do We Need Advanced network research at Fermilab?}

- High-through computing for international particle physics collaborations requires the ability to transport large amount of data quickly around the world

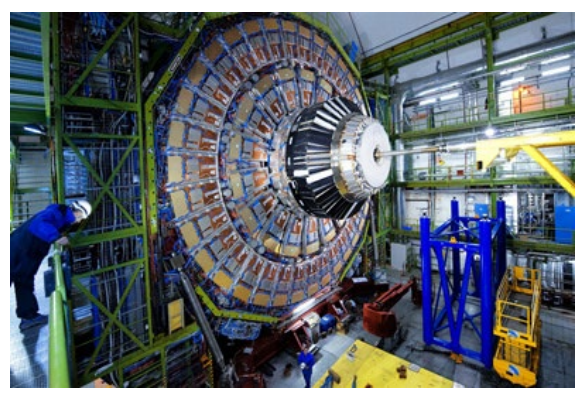

CMS Experiment

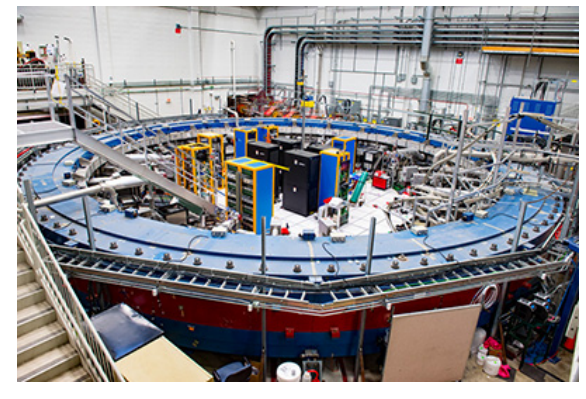

Muons Experiment

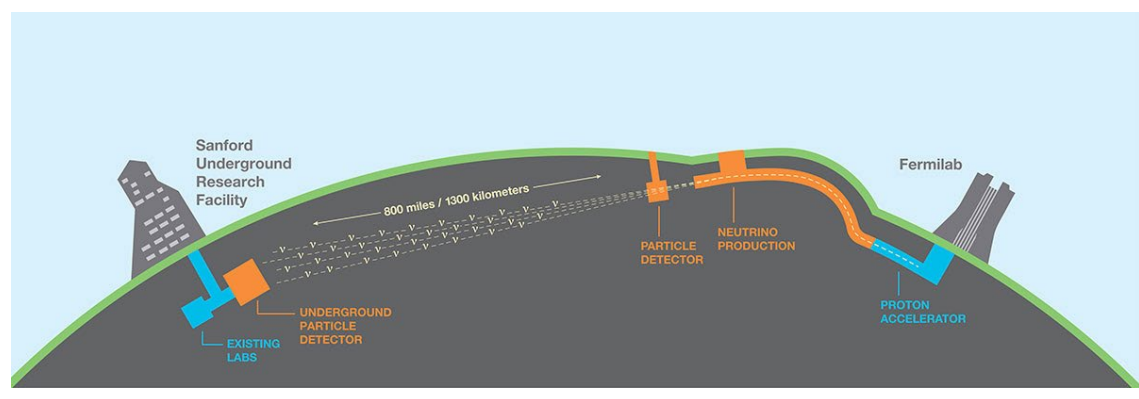

LBNF/DUNE Experiment

- Fermilab has long engaged in network R\&D in support of its mission

- 100-gigabit connectivity to local, national, and international wide-area networks 


\section{Advanced networking facilities at Fermilab}

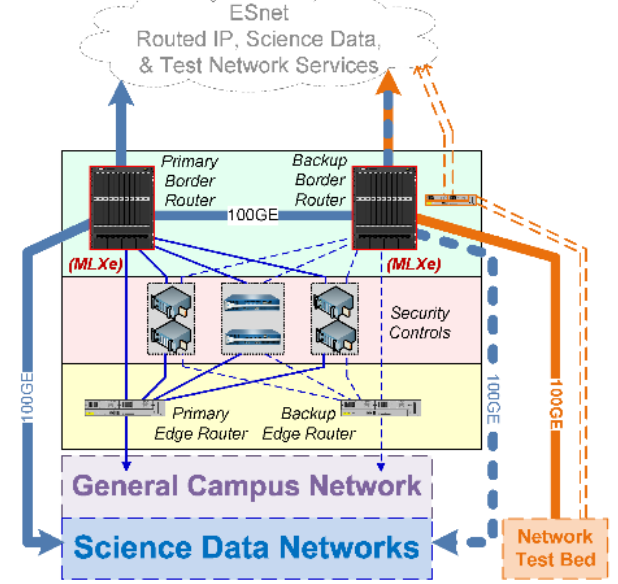

Fermilab Wide Area Networking Facilities

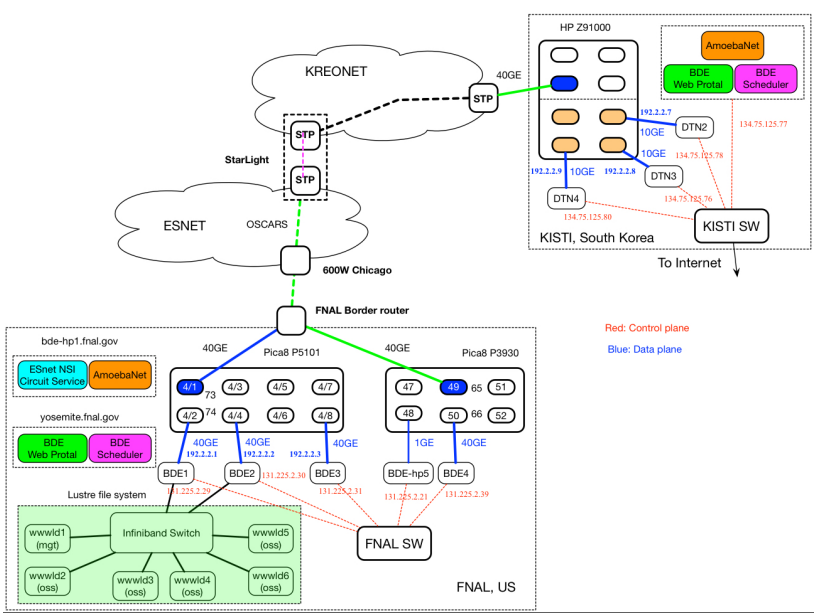

The Cross-Pacific SDN Testbed

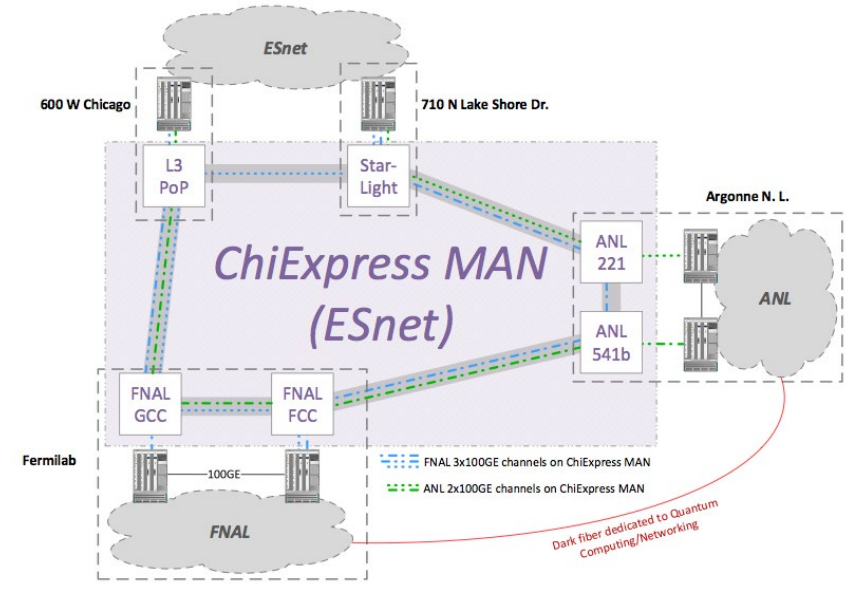

ChiExpress MAN

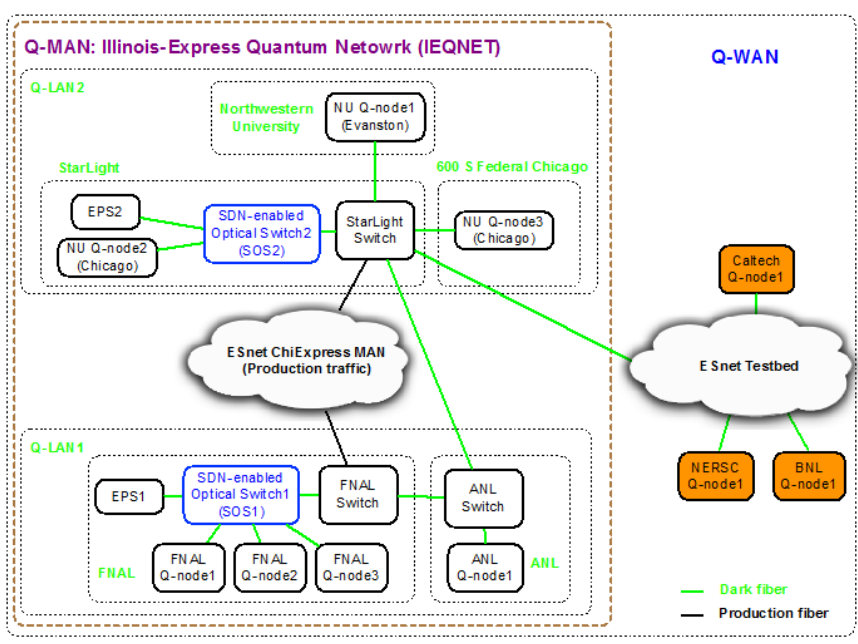

Illinois-Express Quantum Network (IEQNET) 추 Fermilab 


\section{Major Network Research Projects that I lead and/or participate in}

- WireCap: a novel packet capture engine for commodity NICs in high-speed networks

- U.S. Patent 20160127276A1

- http://wirecap.fnal.gov

- mdtmFTP: a high performance data transfer tool

- Funded by DOE ASCR, $\$ 1.5 \mathrm{M}$

- http://mdtm.fnal.gov

- BigData Express

- Funded by DOE ASCR, $\$ 2.2 \mathrm{M}$

- http://bigdataexpress.fnal.gov

- Quantum network research (funded by DOE ASCR, \$3.2M) 


\title{
WireCAP: A Novel Packet Capture Engine for Commodity NICs in High-speed Networks
}

\author{
W. Wu, P. DeMar
}




\section{Packet Capture Engine Basics}

- Capture incoming network data packet for analysis

- Handle a continuous flow of packets

No packet drops!!

- Used by Cyber security and traffic characterization applications

- Implementations found in ASIC, FPGA, and on commodity off-the-shelf (COTS) platforms

- Generic COTS packet capture engine

- Network interface card (NIC) receives network packets

- Packets moved to receive ring buffer

- Packet capture engine provides mechanism(s) to deliver packets from receive ring to application in user space

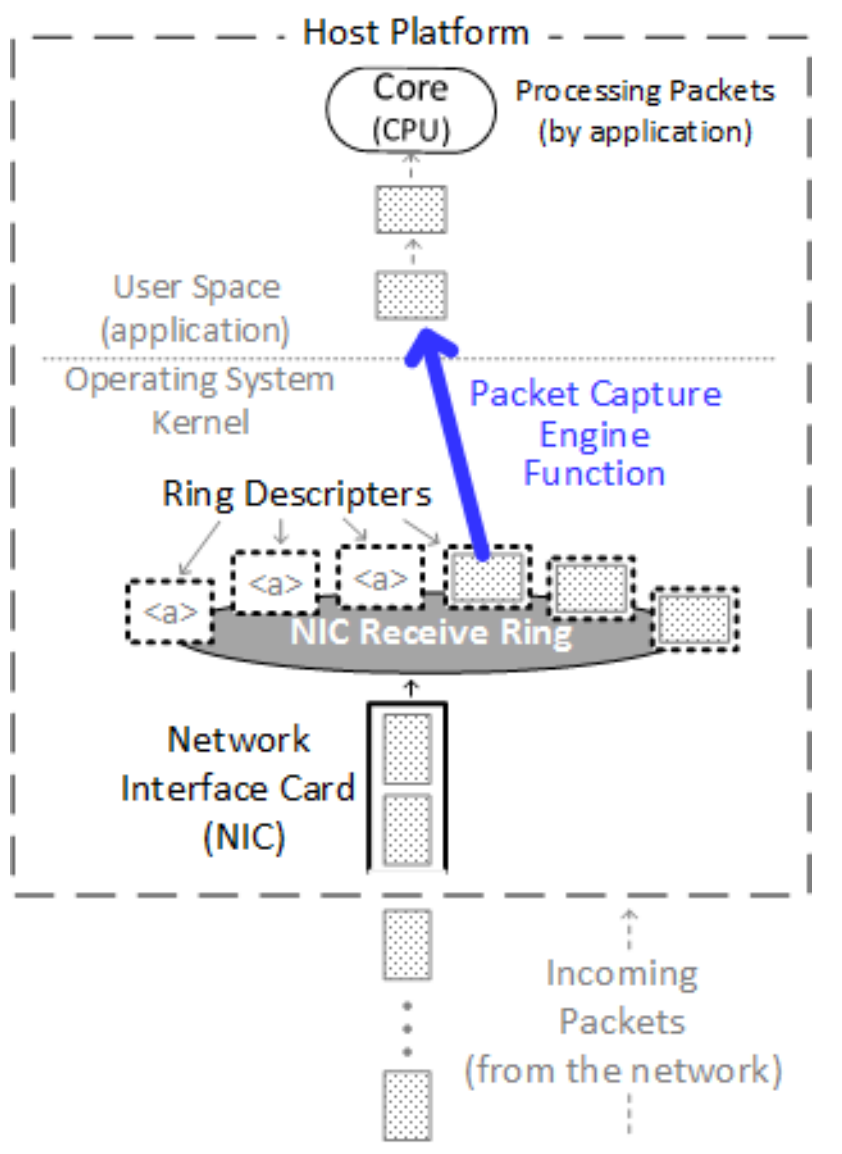




\section{What is WireCAP?}

- An innovative packet capture engine:

- Designed for lossless packet capturing for commodity NICs in high-speed networks

- Cost-effective

- Flexible

- Intended for multicore systems with multi-queue NICs

- Two advanced mechanisms for lossless packet capturing

- Ring-buffer-pool mechanism

- For short-term traffic burstiness

- Buddy-group mechanism

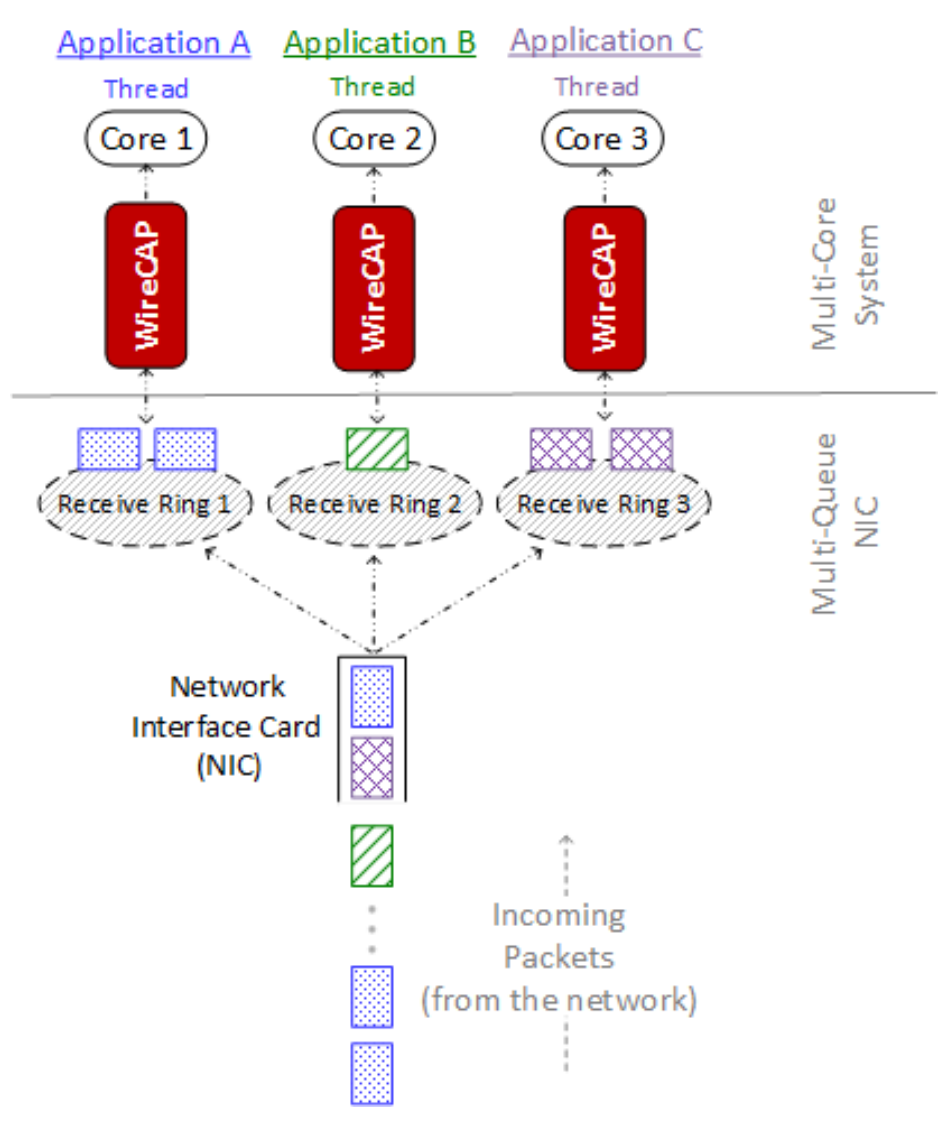

- For long-term traffic flooding 


\section{Miscellaneous}

- Project website: http://wirecap.fnal.gov

- Paper

- Wenji Wu, Phil DeMar, "WireCAP: a Novel Packet Capture Engine for Commodity NICs in High-speed Networks," IMC'14, November 05 - 07 2014, Vancouver, BC, Canada.

- Patent

- U.S. Patent 20160127276A1, filed November 4, 2015, and issued September 18, 2018.

- Issued to multiple agencies, including

- U.S. Army Research Lab, U.S. Naval Research Lab, U.S. Air Force Research Lab, LANL, ... 


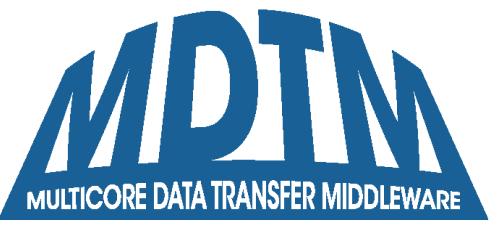

\title{
mdtmFTP: a high-performance data transfer tool
}

\author{
W. Wu, L. Zhang, P. DeMar, L. Carpenter
}




\section{mdtmFTP: a high-performance data transfer tool}

- Pipelined I/O-centric design to streamline data transfer

- Multicore-aware data transfer middleware (MDTM) optimizes use of underlying multicore system

- Extremely efficient in transferring of Lots of Small Files (LOSF)

- Various optimization mechanisms

- Zero copy

- Asynchronous I/O

- Batch processing

\section{A DOE/SC/ASCR-sponsored research project Software is available at: http://mdtm.fnal.gov}

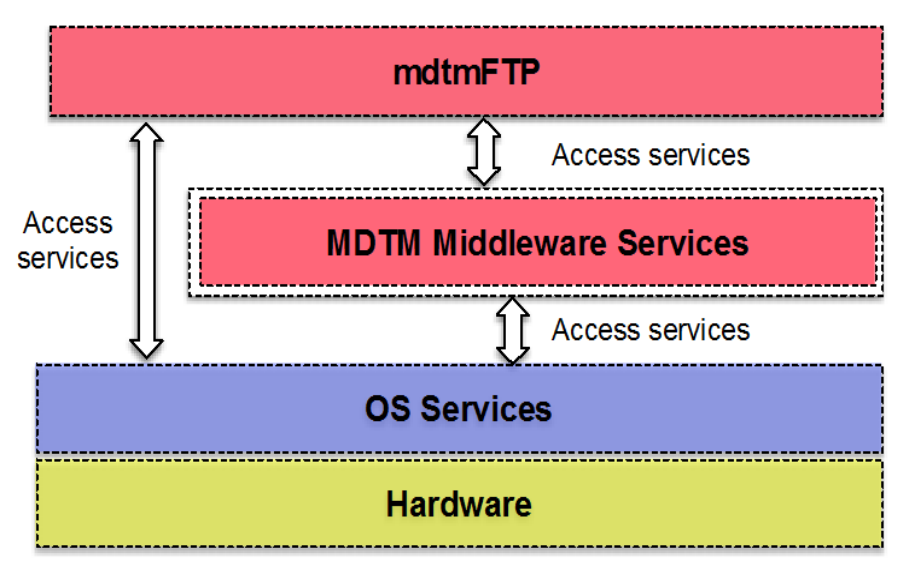

출 Fermilab 


\section{mdtmFTP - A pipelined I/O centric design}

- Dedicated I/O threads to perform network/disk I/Os in parallel

- MDTM middleware to schedule cores for $1 / O$ threads

- Advanced data buffer mechanism to improve $\mathrm{I} / \mathrm{O}$ performance

- Data transfers are executed in a pipelined manner

- A data transfer task is split into multiple subtasks

- Subtasks are executed in parallel

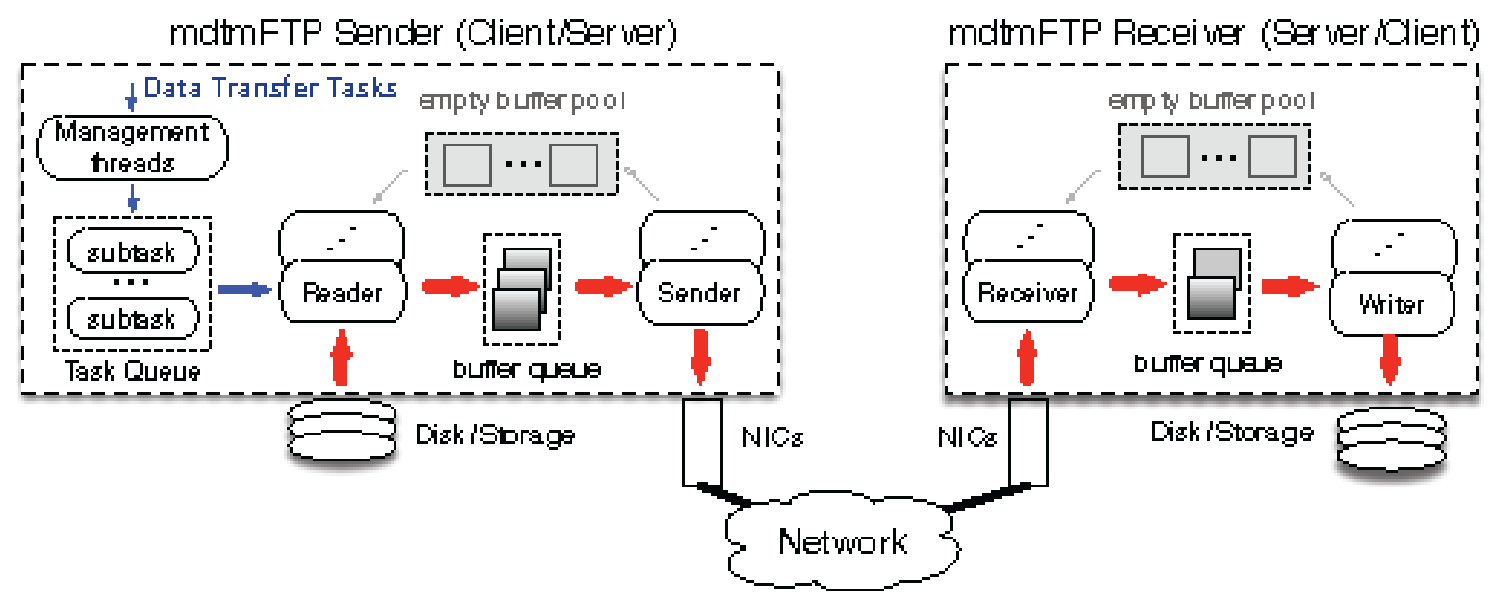




\section{mdtmFTP - A large virtual file transfer mechanism}

- Treat a dataset as a large "virtual file".

- Each file in the dataset is treated as a file segment in the virtual file, and sequentially "added" to the virtual file.

- The virtual file is logically, instead of physically, created.

- Different than Tar-based solutions

- The whole data set is transferred continuously and seamlessly as a single virtual file.

- Different than GridFTP's per-file-based mechanisms (e.g., pipelining, concurrency)

- Avoid protocol processing on a per-file basis

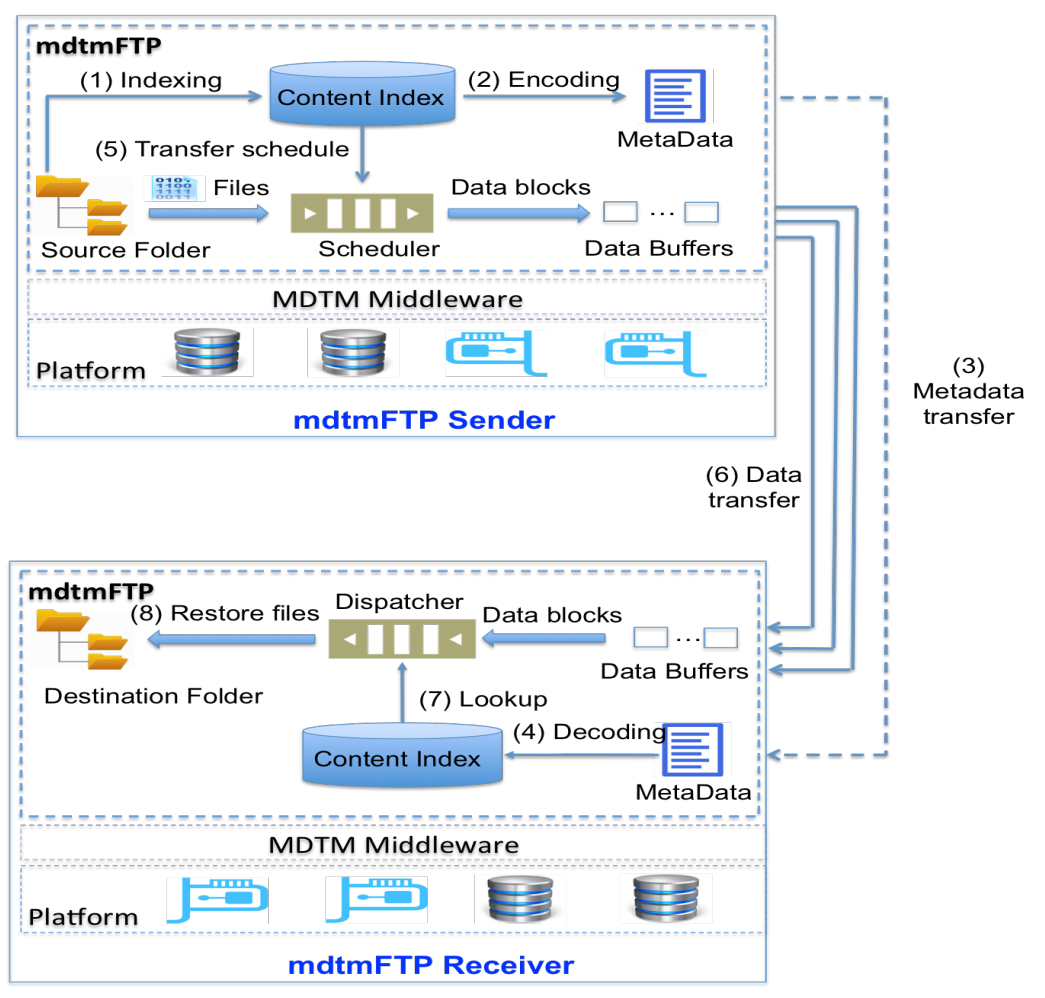

- Allow for batch processing small files in the sender/receiver to optimize I/O performance 


\section{LSST SC'18 News Release}
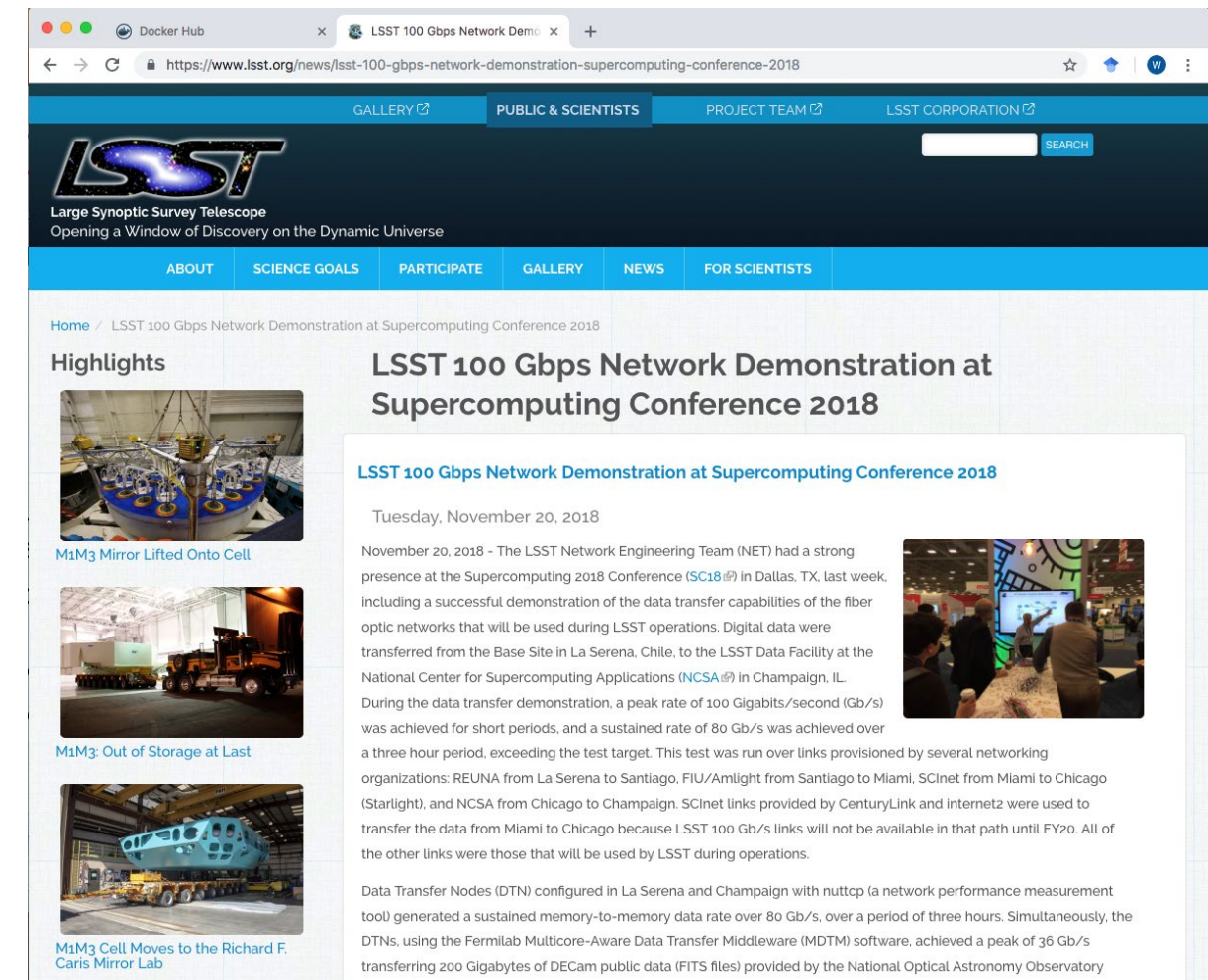

See all

Tuesday. November 20, 2018
LSST 100 Gbps Network Demonstration at

Supercomputing Conference 2018

LSST 100 Gbps Network Demonstration at Supercomputing Conference 2018

November 20.2018 - The LSST Network Engineering Team (NET) hada stong presence at the Supercomputing 2018 Conference (SC18 \&) in Dallas. Tx. last week.

cessful demonstration of the data transfer capabilities of the fiber

opltcher will be used during LSST operations. Digital data were National Center for Supercomputing Applications (NCSA During the data transfer demonstration, a peak rate of 100 Gigabits/second (Gib/s)

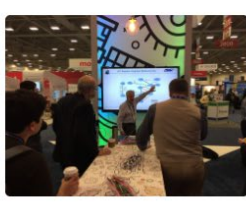

was achieved for short periods, and a sustained rate of $80 \mathrm{~Gb} / \mathrm{s}$ was achieved

oned by several networking organizatons. REUNA from La Serena to Santiago, FIU/Amlight from Santiago to Miami. SCInet from Mlami to chicago tStangh the other links were those that will be used by LSST during operations

Data Transfer Nodes (DTN) connigured in La Serena and Champaign with nutcp ta network periormance measurement. tooll generated a sustained memory-to-memory data rate over $80 \mathrm{~Gb} / \mathrm{s}$, over a period of three hours. Simultaneously, the DTNs, using the Fermilab Multicore-Aware Data Transfer Middleware (MDTM) software, achieved a peak of $36 \mathrm{~Gb} / \mathrm{s}$ transferring 200 Gigabytes of DECam public data (FITS files) provided by the National Optical Astronomy Observatory (NOAOAs). Note that in LSST operations, there will be over 20 DTNS (aka archiver/forwarders) simultaneously sending data so each one will require far less than $36 \mathrm{~Gb} / \mathrm{s}$. In addition, on the Champaign end the flies were ingested into a GPFS shared file system, and a Jupyter Notebook running an application provided by LSST Data Management was used to visualize the files Finally, an additional test transfer from Champaign to La Serena is being conducted and has so far achieved a peak of $40 \mathrm{~Gb} / \mathrm{s}$. sufficient for the annual transfer of LSST Data Releases to Chile.

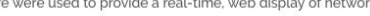
performance durng the demonstration. This was monitored Iive from the NCSA booth at the Supercomputing 2018 Conference. A number of cont
question and answer session.

According to LSST NET Lead Jeff Kantor. "This demonstration shows not only that we have con from
"Simultaneously, the DTNs, using the Fermilab MulticoreAware Data Transfer Middleware (MDTM) software, achieved a peak of $36 \mathrm{~Gb} / \mathrm{s}$ transferring 200 Gigabytes of DECam public data (FITS files) provided by the National Optical Astronomy Observatory." 


\section{mdtmFTP Statistics}

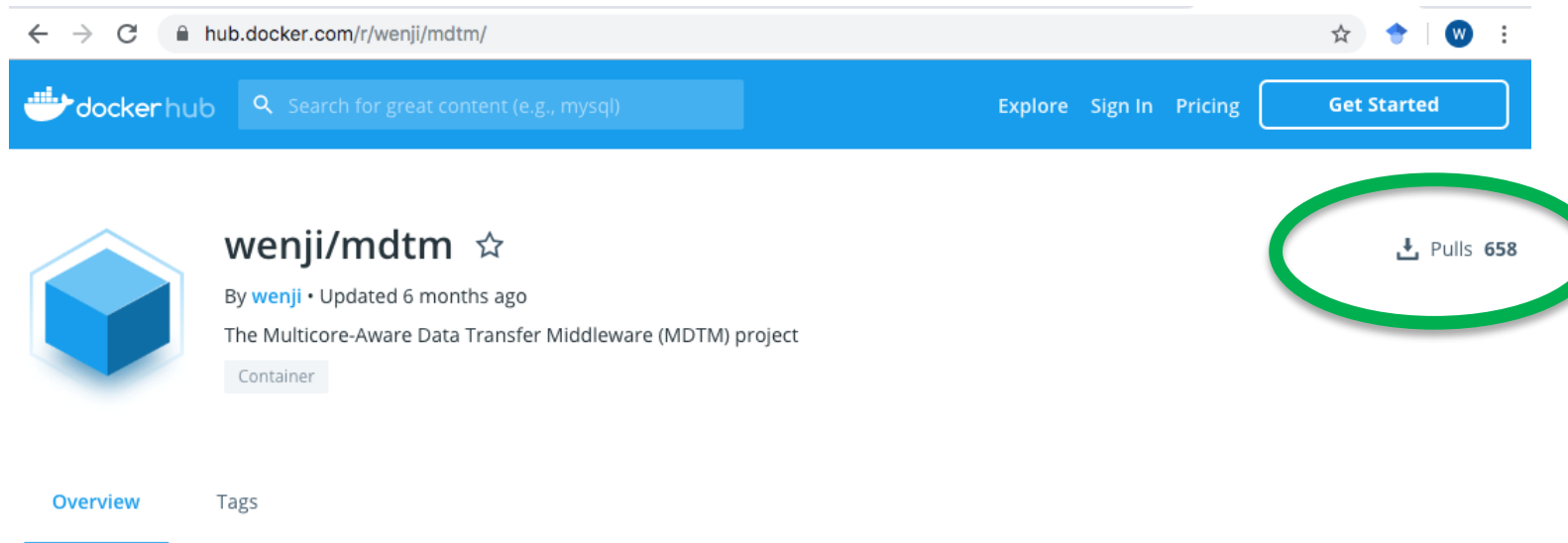

Docker Pull Command

The MDTM project is funded by DOE's Advanced Scientific Computing Research (ASCR) office. MDTM aims to accelerate data movement toolkits on multicore systems.

The MDTM project website: http://mdtm.fnal.gov

mdtmGUI: a web-based tool developed to monitor and manage MDTM-enabled DTNs.

mdtmFTP: a high-performance data transfer tool for multicore systems. The Admin manual is available at https://mdtm.fnal.gov/downloads/manual/index.htm

If you want to report bugs,ask questions, suggest improvements, or request features, please send emails to wenji@fnal.gov, liangz@fnal.gov.

The latest mdtmFTP docker container is released on Feb. 19, 2019.

- latest, most recent mdtmFTP docker image, released on Feb. 19, 2019

- 1.0.3, mdtmFTP docker image released 1.0.3, Feb. 19, 2019

\section{docker pul1 wenji/ndtm $\square$}

Owner

wenji

\section{8 downloads}
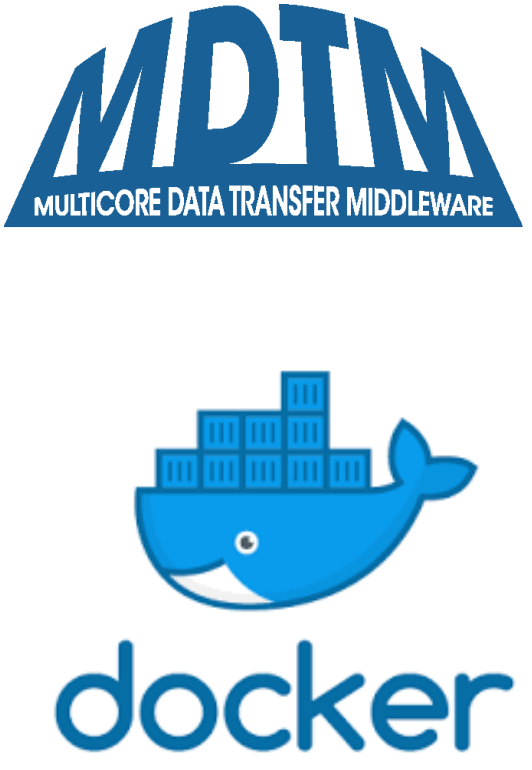

\section{https://hub.docker.com/r/wenji/mdtm/}




\section{BigData Express}




\title{
Many people's hard work
}

\author{
FNAL: $\quad$ Qiming Lu, Liang Zhang, Sajith Sasidharan, \\ Wenji Wu, Phil DeMar
}

ESnet: $\quad$ Chi Guok, John Macauley, Inder Monga

iCAIR/StarLight: Se-young Yu, Jim-Hao Chen, Joe Mambretti

KISTI:

Jin Kim, Seo-Young Noh

UMD/MAX: $\quad$ Xi Yang, Tom Lehman 


\section{Data Transfer Challenges in BigData Era}

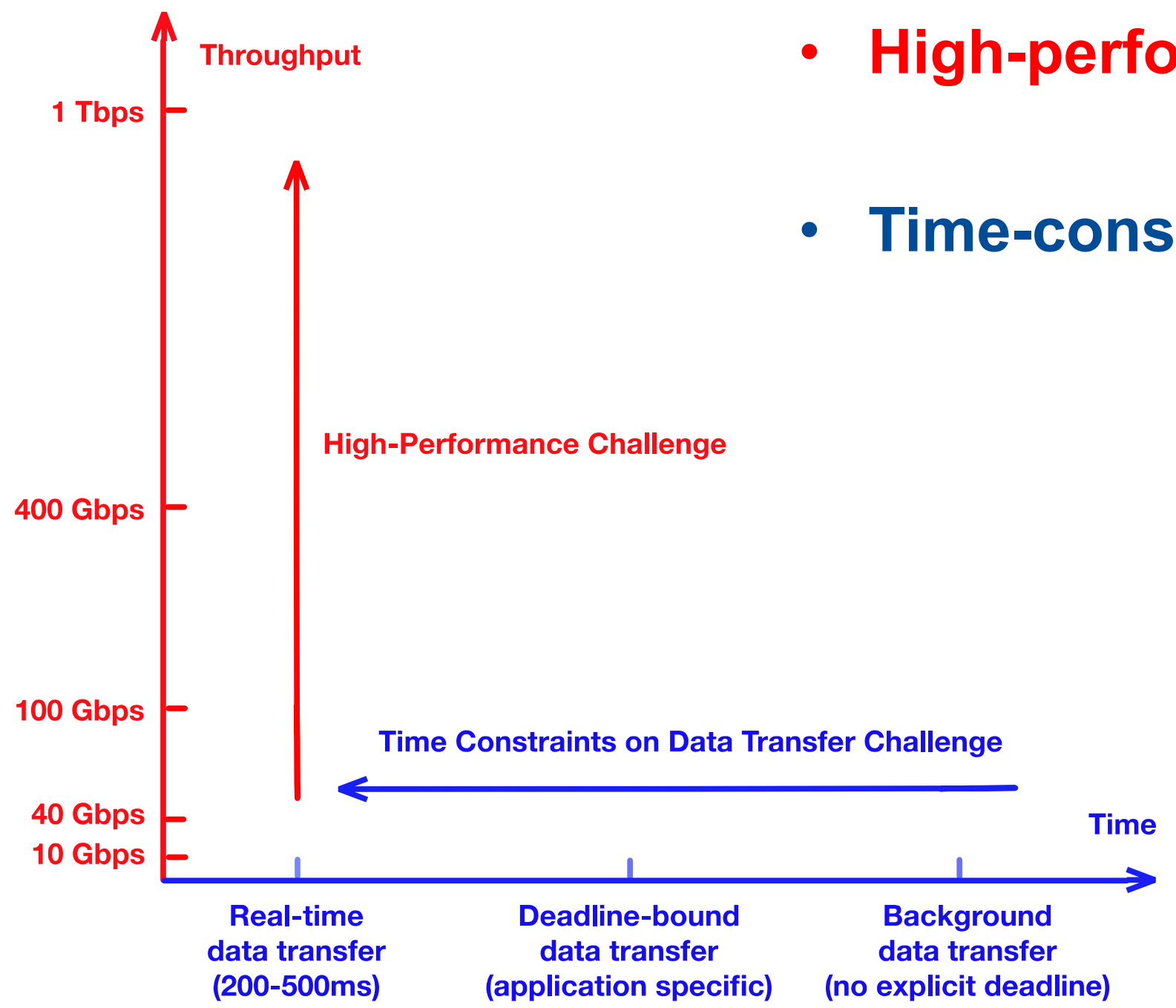




\section{Data Transfer - State of the Art}

- Advanced data transfer tools and services developed

- GridFTP, BBCP

- PhEDEx, LIGO Data Replicator, Globus Online

- Numerous enhancements

- Parallelism at all levels

- Multi-stream, Multicore, Multi-path parallelism

- Science DMZ architecture

- Terabit networks 


\section{Problems with Existing Data Transfer Tools \& Services}

- Disjoint end-to-end data transfer loop

- Cross-interference between data transfers

- Oblivious to user requirements (e.g., deadlines and QoS requirements)

- Inefficiencies arise with existing data transfer tools running on DNTs 


\section{Problem 1 - Disjoint end-to-end data transfer loop}

- Distributed resource management model

- Resource contention

- Performance mismatch
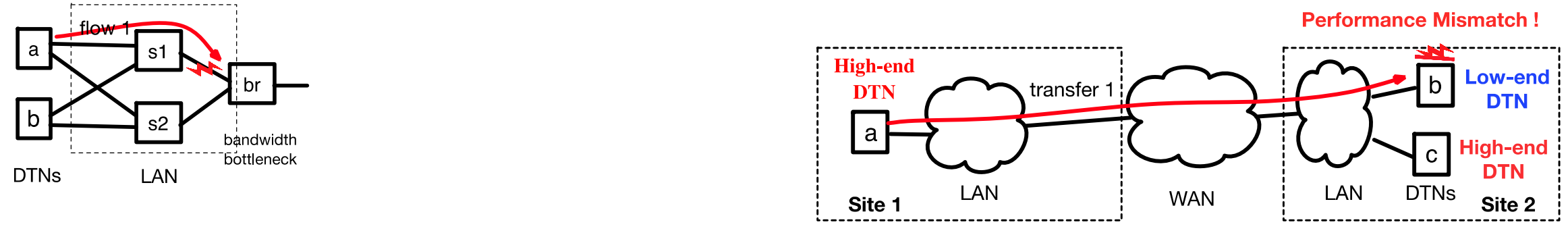

a. without coordination
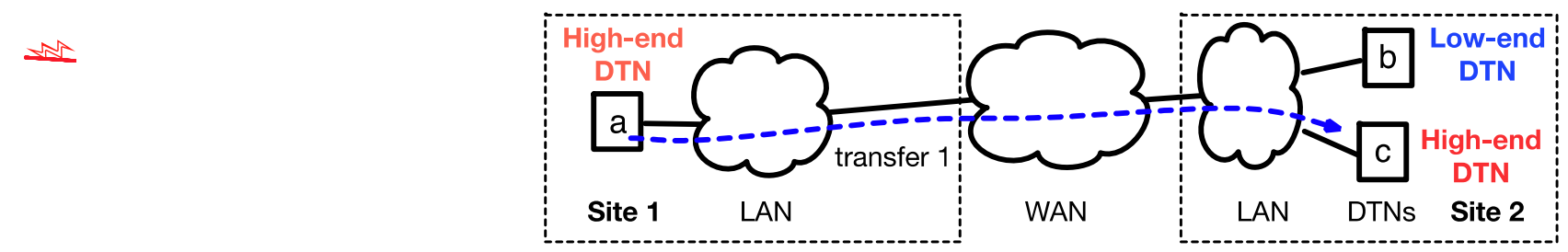

b. with coordination 


\section{Problem 2 - Cross-interference between data transfers}

\begin{tabular}{|c|c|c|}
\hline $\begin{array}{c}\text { Severe } \\
\text { Cross-interference }\end{array}$ & $\begin{array}{c}\text { Resource } \\
\text { Contention }\end{array}$ & $\begin{array}{c}\text { Poor } \\
\text { Performance }\end{array}$ \\
\cline { 2 - 3 }
\end{tabular}

- Degraded performance

- High variability in data transfer performance 


\section{Problem 3 - Oblivious to user requirements}

- Data transfer jobs are schedule on a first-come, first serve basis

- Without deadline awareness

- Resources are shared fairly among data transfer jobs

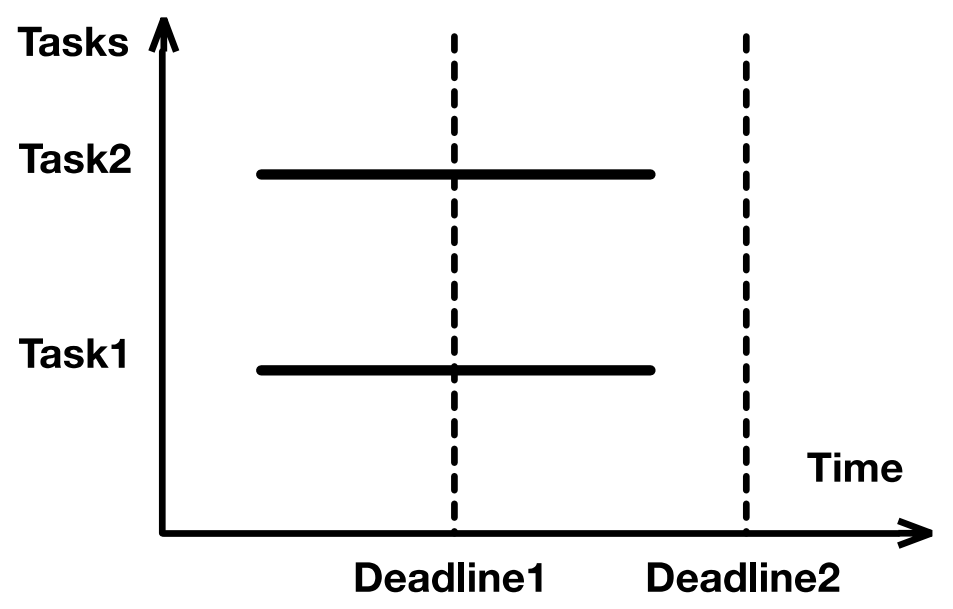

a. without deadline awareness

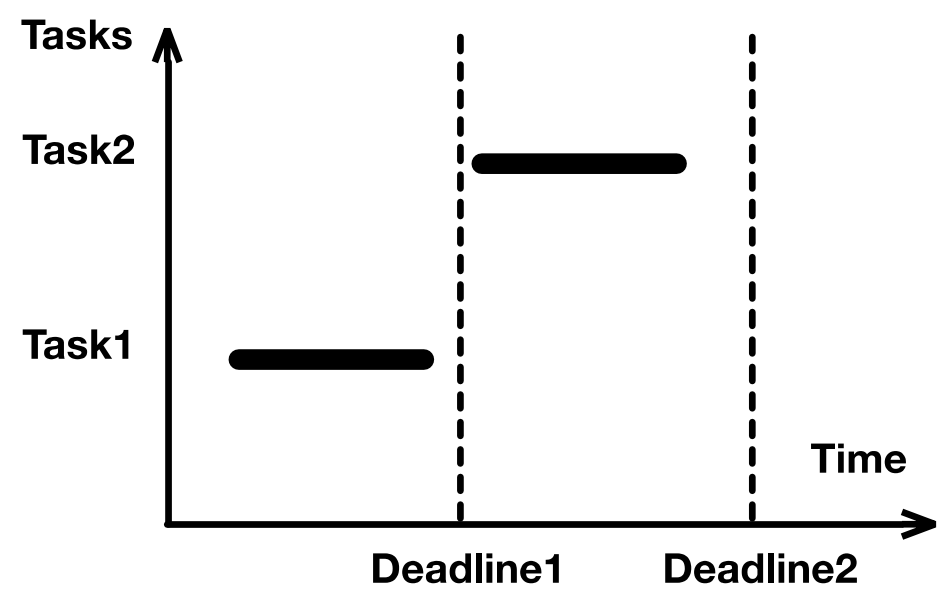

b. with deadline awareness 


\section{Problem 4 - Inefficiencies arises when existing data transfer tools run on DTNs}

- I/O locality on NUMA systems

- Cache thrashing

- Scheduling overheads

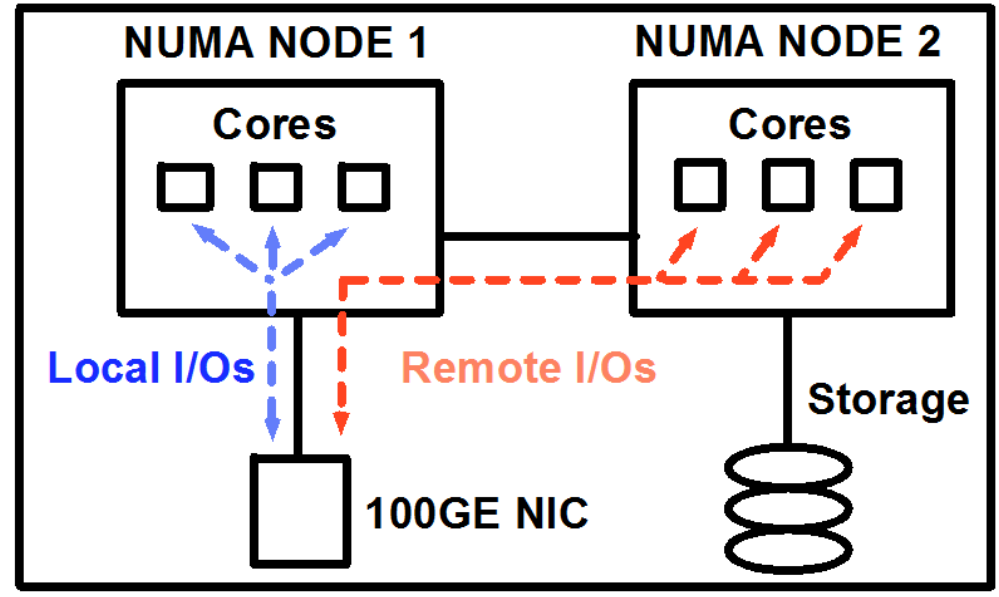

\section{Need high-performance data transfer tool!}




\section{Our Solution - BigData Express}

- BigData Express: a schedulable, predictable, and highperformance data transfer service

- A peer-to-peer, scalable, and extensible data transfer model

- A visually appealing, easy-to-use web portal

- A high-performance data transfer engine

- A time-constraint-based scheduler

- On-demand provisioning of end-to-end network paths with guaranteed QoS

- Robust and flexible error handling

- ClLogon-based security 


\section{BigData Express Major Components}

- BigData Express Web Portal

$>$ Access to BigData Express services

- BigData Express Scheduler

$>$ Time-constraint-based scheduler

$>$ Co-scheduling DTN, storage, \& network

- AmoebaNet

$>$ Network as a service

$>$ Rate control

- mdtmFTP

$>$ High-performance data transfer engine

$>$ http://mdtm.fnal.gov

- Data Transfer Launching Agent

$>$ Launch data transfer jobs

$>$ Support different data transfer protocols
- DTN Agent

$>$ Manage and configure DTNs

$>$ Collect \& report DTN configuration and status

- Storage Agent

$>$ Manage and configure storage systems

$>$ I/O estimation 


\section{BigData Express -- Distributed}

\section{Software Stack@ a BigData Express Site}

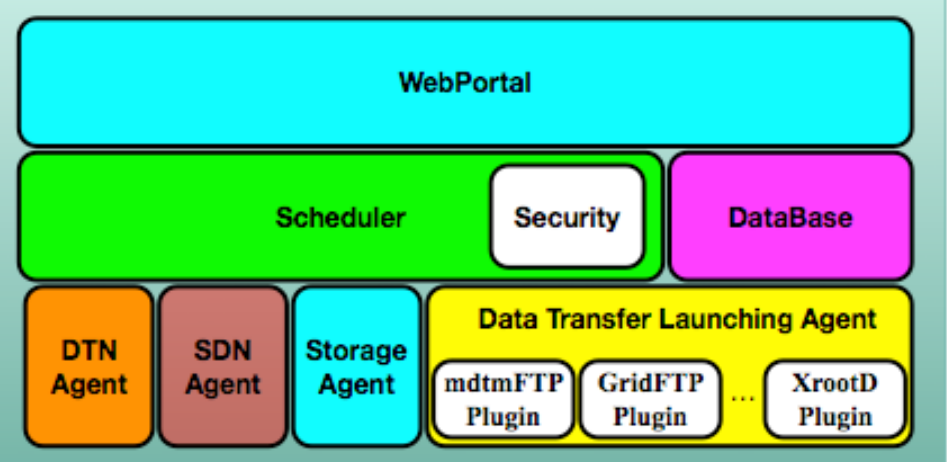

A Peer-to-Peer model
BigData Express site

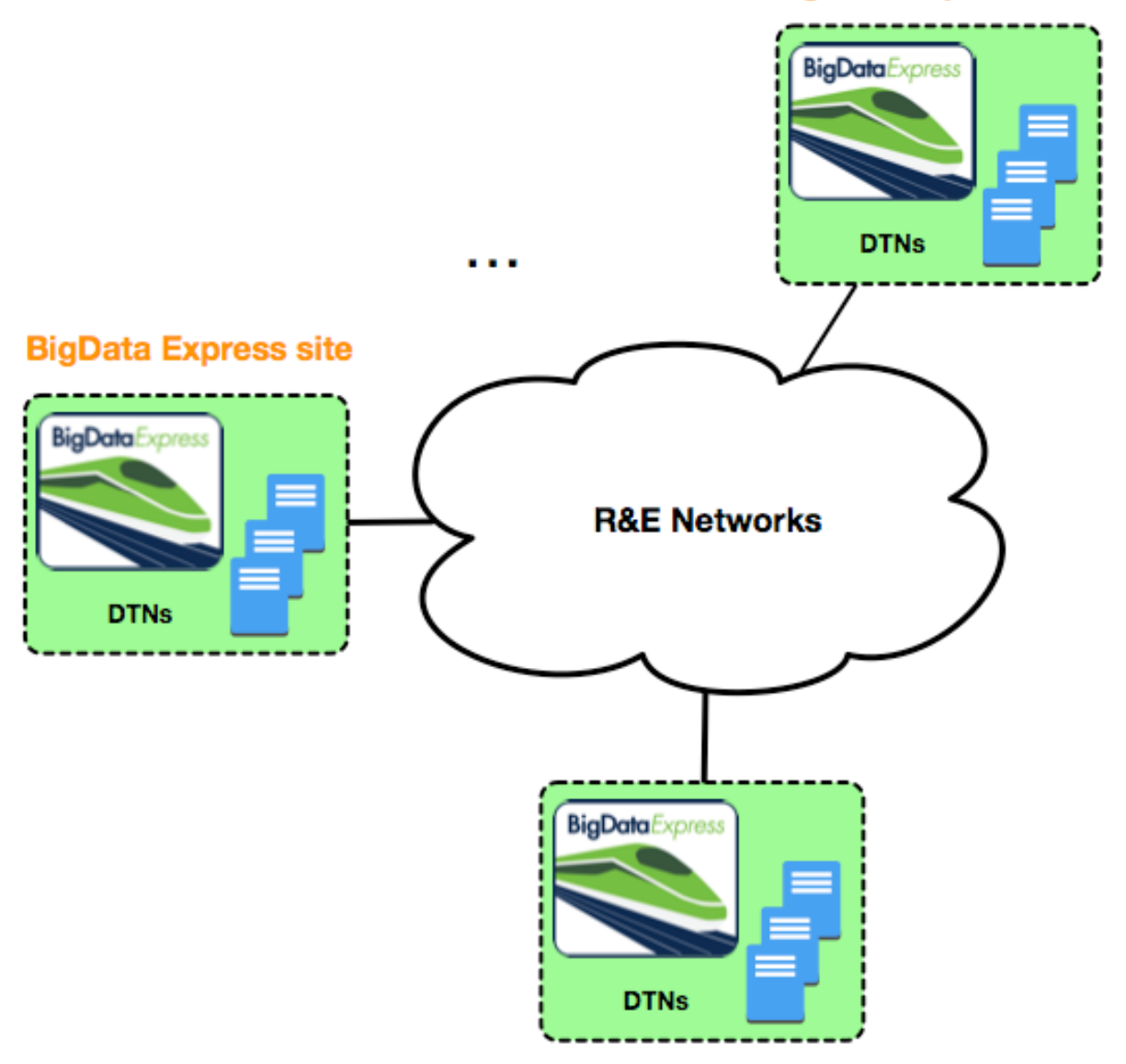

BigData Express site 


\section{BigData Express -- Flexible}

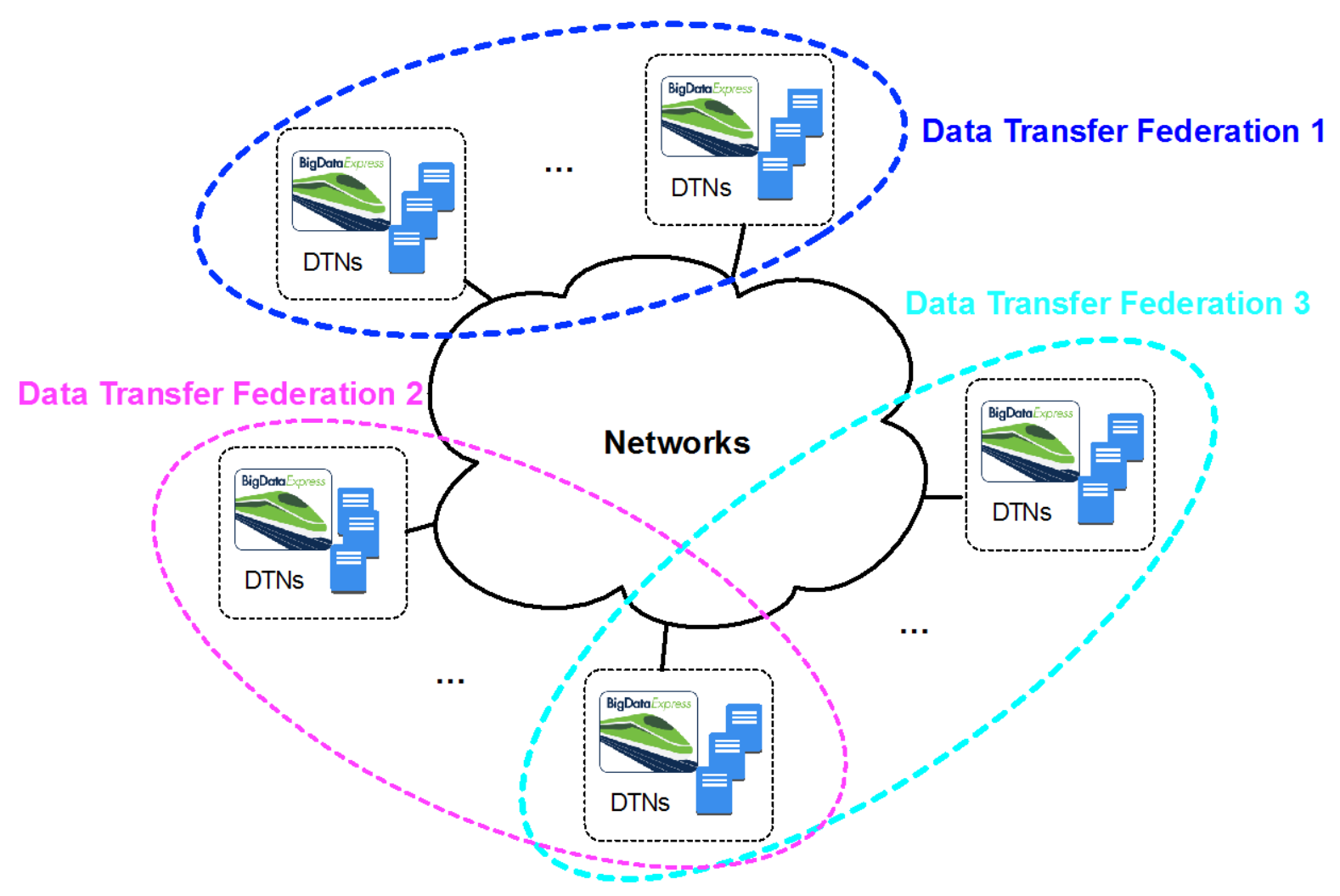

- Flexible to set up data transfer federations

- Providing inherent support for incremental deployment 


\section{BigData Express - Scalable}

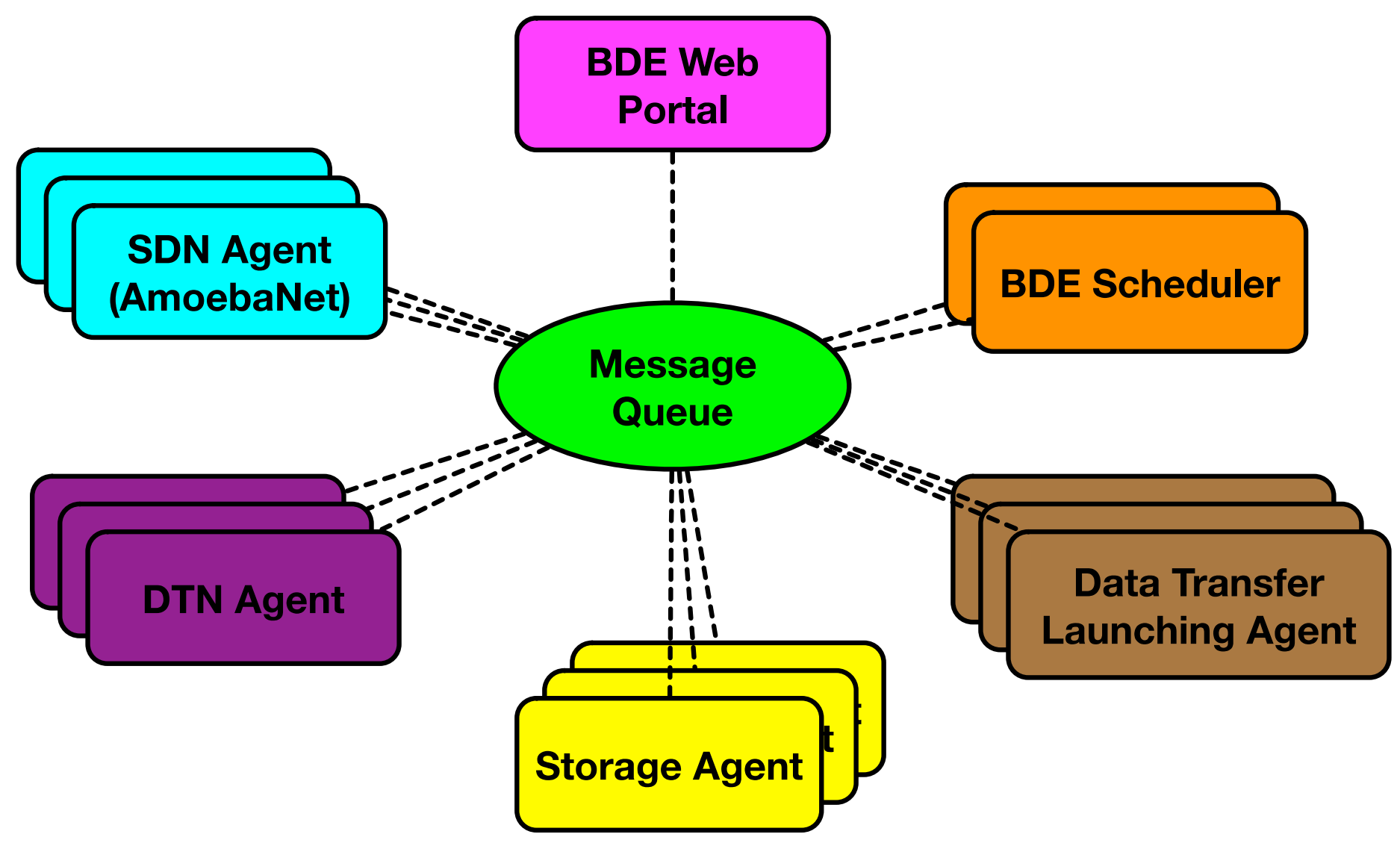

- BigData Express scheduler manages site resources through agents - Use MQTT as message bus 


\section{BigData Express - Extensible}

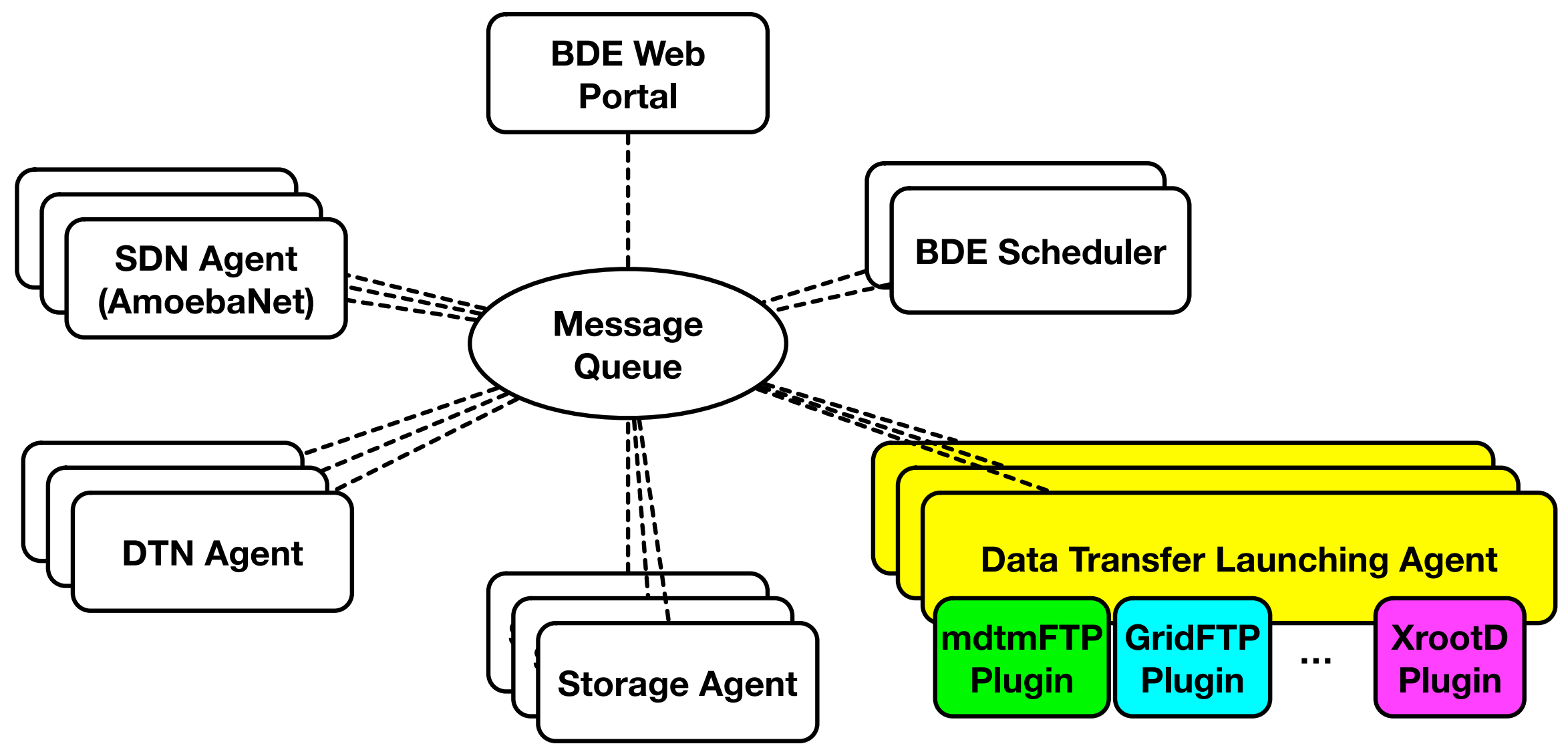

- Extensible plugin framework to support various data transfer protocols

- mdtmFTP, GridFTP, XrootD, ... 


\section{BigData Express - End-to-End Data Transfer Model}

Site A - Smart E2E

Data Transfer Orchestrator

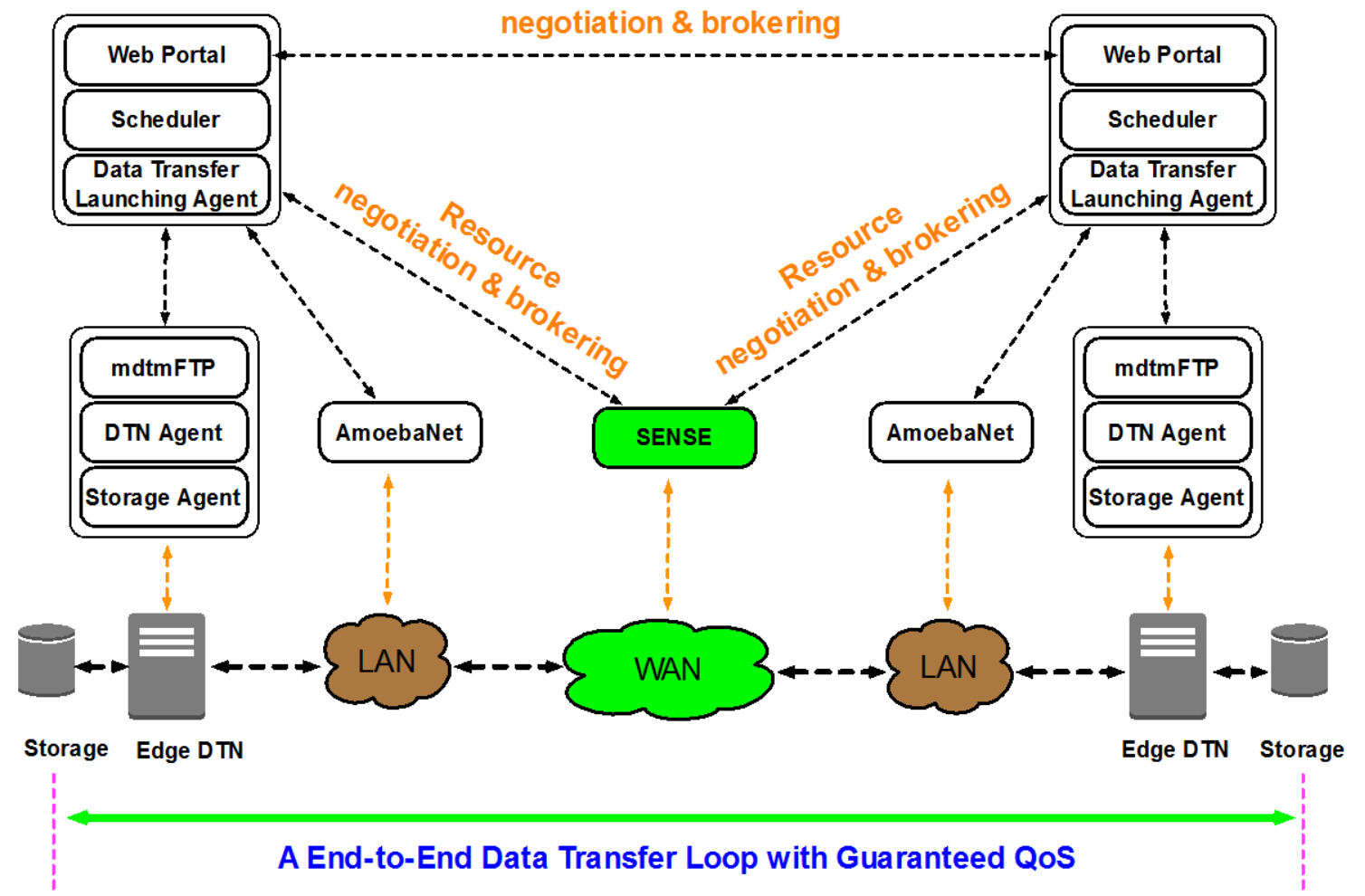

Site B - Smart E2E

Data Transfer Orchestrator

- Application-aware network service

- Fast-provisioning of end-to-end network paths with guaranteed QoS

- Distributed resource negotiation \& brokering 


\section{BigData Express - High Performance Data Transfer}

\begin{tabular}{|l|c|c|c|c|}
\hline & moltmFTP & FDT & GridFTP & BBCP \\
\hline Large file data transfer $(\mathbf{1} \times \mathbf{1 0 0 G})$ & 74.18 & 79.89 & 91.18 & Poor \\
\hline Folder data transfer $(\mathbf{3 0} \times \mathbf{1 0 G})$ & 192.19 & $\mathbf{2 1 7}$ & $\mathbf{3 2 0 . 1 7}$ & Poor \\
\hline Folder data transfer (Linux 3.12.21) & 10.51 & - & 1006.02 & Poor \\
\hline
\end{tabular}

Time-to-completion (Seconds) - Client/Server mode Lower is better

\begin{tabular}{|l|c|c|c|c|}
\hline & mottmFTP & FDT & GridFTP & BBCP \\
\hline Large file data transfer $(\mathbf{1} \times \mathbf{1 0 0 G})$ & 34.976 & N/A & 106.84 & N/A \\
\hline Folder data transfer $(\mathbf{3 0} \times \mathbf{1 0 G})$ & 95.61 & N/A & - & N/A \\
\hline Folder data transfer (Linux 3.12.21) & 9.68 & N/A & - & N/A \\
\hline
\end{tabular}

Time-to-completion (Seconds) - 3rd party mode

Lower is better

Note 1: "-" indicates inability to get transfer to work

Note 2: BBCP performance is very poor, we do not list its results here

Note 3: BBCP and FDT support $3^{\text {rd }}$ party data transfer. But BBCP and FDT couldn't run $3^{\text {rd }}$ party data transfer on ESNET testbed due to testbed limitation

mdtmFTP is faster than existing data transfer tools, ranging from $8 \%$ to $9500 \%$ ! @ESnet 100GE SDN Testbed, 


\section{BigData Express - High Performance Data Transfer}

\section{$S T$ 浓 $R$ R I I GHTS T}

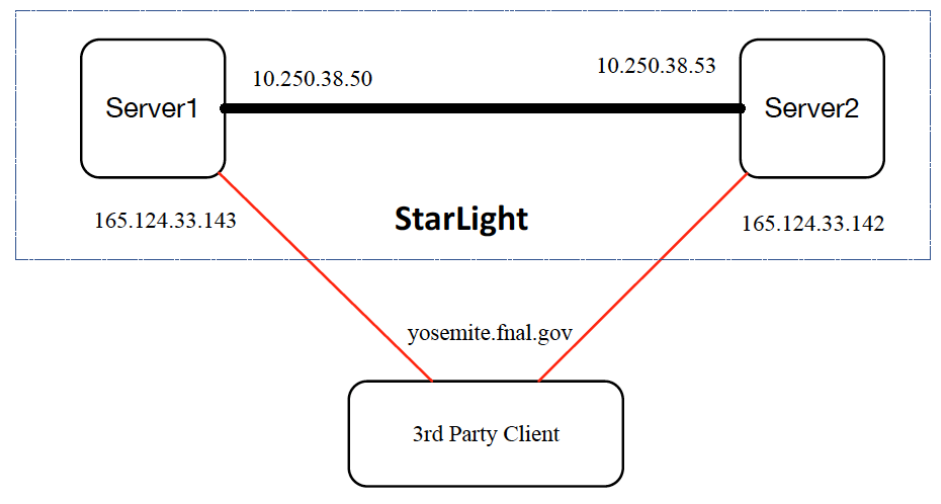

StarLight 100GE Testbed

\section{Performance - Aggregate throughput}

\begin{tabular}{|l|l|l|l|l|}
\hline $\mathrm{Gb} / \mathrm{s}$ & Run1 & Run2 & Run4 & Run8 \\
\hline GridFTP & $6.2 \mathrm{Gbps}$ & $12.24 \mathrm{Gbps}$ & $20.35 \mathrm{Gbps}$ & $28.32 \mathrm{Gbps}$ \\
\hline mdtmFTP & $13.27 \mathrm{Gbps}$ & $23.80 \mathrm{Gbps}$ & $28.354 \mathrm{Gbps}$ & $43.94 \mathrm{Gbps}$ \\
\hline
\end{tabular}

mdtmFTP vs. GridFTP

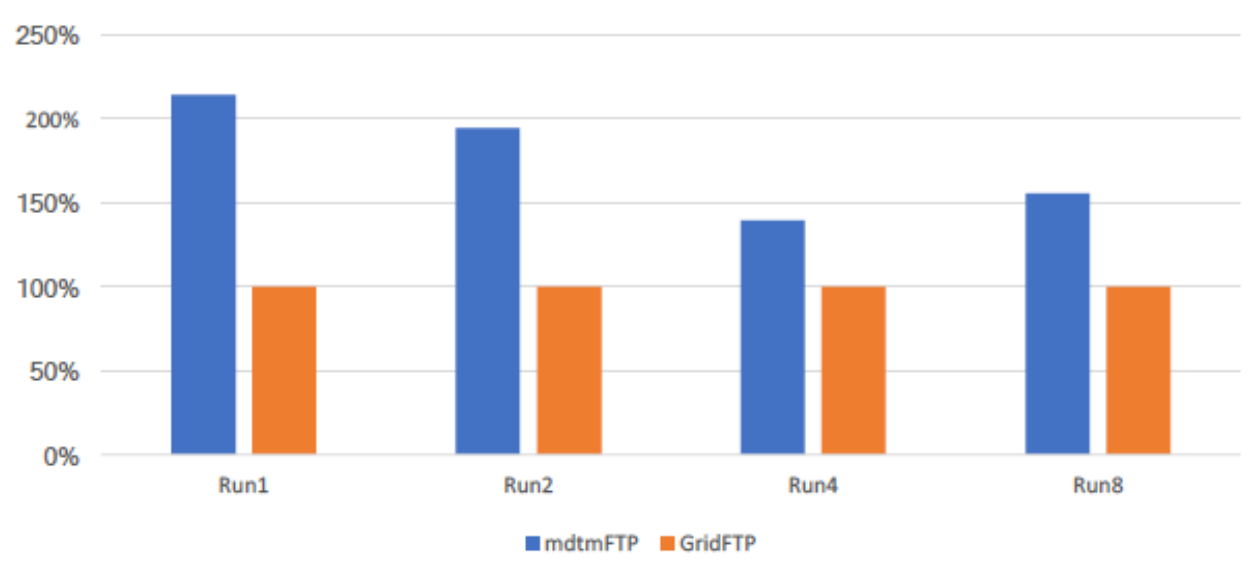

mdtmFTP is faster than GridFTP, ranging from $40 \%$ to $114 \%$ ! @StarLight 100GE Testbed 


\section{BigData Express - Mechanism Summary}

\section{Problems with existing data transfer tools BigData Express Solutions}

- Distributed resource negotiation \& brokering

- Disjoint end-to-end data transfer loop

- Co-scheduling of DTN, storage, \& networking

- On-demand provisioning of end-to-end network path with guaranteed QoS

- Time-constraint-based scheduler

- Cross-interference between data transfers

- Admission control

- Rate control

- Time-constraint-based scheduler

- Three classes of data transfer

- Oblivious to user requirements

- Inefficiencies arises when existing data transfer tools run on DTNs

- $\quad$ dtmFTP - A high-performance data transfer engine 


\section{BigData Express vs. Globus Online}

\begin{tabular}{|c|c|c|}
\hline Features & BigData Express & Globus Online \\
\hline Architecture & $\begin{array}{l}\text { - } \quad \text { Distributed service } \\
\text { - } \quad \text { Flexible to set up data transfer federations }\end{array}$ & - Centralized service \\
\hline $\begin{array}{l}\text { Supported } \\
\text { Protocols }\end{array}$ & $\begin{array}{l}\text { - Extensible plugin framework to support } \\
\text { multiple protocols: } \\
\circ \text { mdtmFTP } \\
\circ \text { GridFTP, XrootD, SRM (coming soon) }\end{array}$ & - GridFTP \\
\hline SDN Support & $\begin{array}{l}\text { - Yes, Network as a service } \\
\text { - Fast-provisioning end-to-end network paths } \\
\text { with guaranteed QoS }\end{array}$ & - Not in production \\
\hline $\begin{array}{l}\text { Supported Data } \\
\text { Transfers }\end{array}$ & $\begin{array}{l}\text { - } \text { Real-time data transfer } \\
\text { - } \text { Deadline-bound data transfer } \\
\text { - } \text { Best-effort data transfer }\end{array}$ & - Best-effort data transfer \\
\hline Error Handling & $\begin{array}{l}\text { - } \quad \text { Checksum } \\
\text { - } \quad \text { Retransmit }\end{array}$ & $\begin{array}{ll}\text { - } & \text { Checksum } \\
\text { - } & \text { Retransmit }\end{array}$ \\
\hline
\end{tabular}




\section{BigDataExpress \\ BigData Express SC18 DEMO}

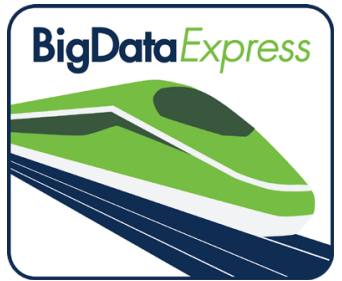

\section{Fermilab}
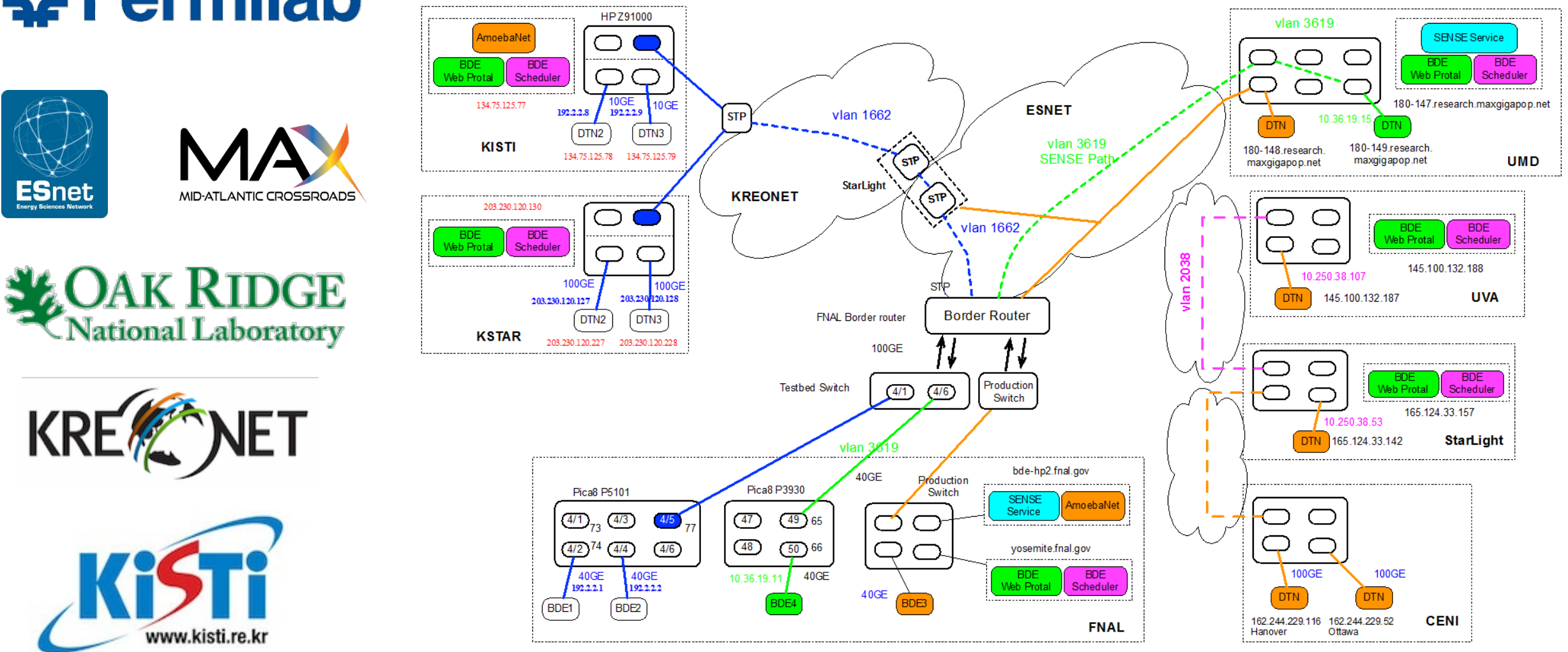

\section{SURF NET




\section{BigData Express - Deployment}

- Asia

- KISTI, South Korea

- KSTAR

- Europe

- University of Amsterdam, Netherlands

- CERN (coming soon)

- North America

- Fermilab

- StarLight, Northwestern University

- UMD/MAX, University of Maryland, College Park

- Ciena (Canada)

- US East

- CA East

- Australia \& Pacific areas

- National Computational Infrastructure (NCI)

- PAWSEY supercomputing center (coming soon)

SURF NET $D$

\section{Fermilab}

$S T$ 沵 $R$ L I GHTS

MAY

ciena.

Experience. Outcomes.

$\mathrm{NCl}$

Z) PAWSEY

贲Fermilab 


\section{Quantum Network Research}

\section{\#Fermilab}




\section{Quantum Network Research}

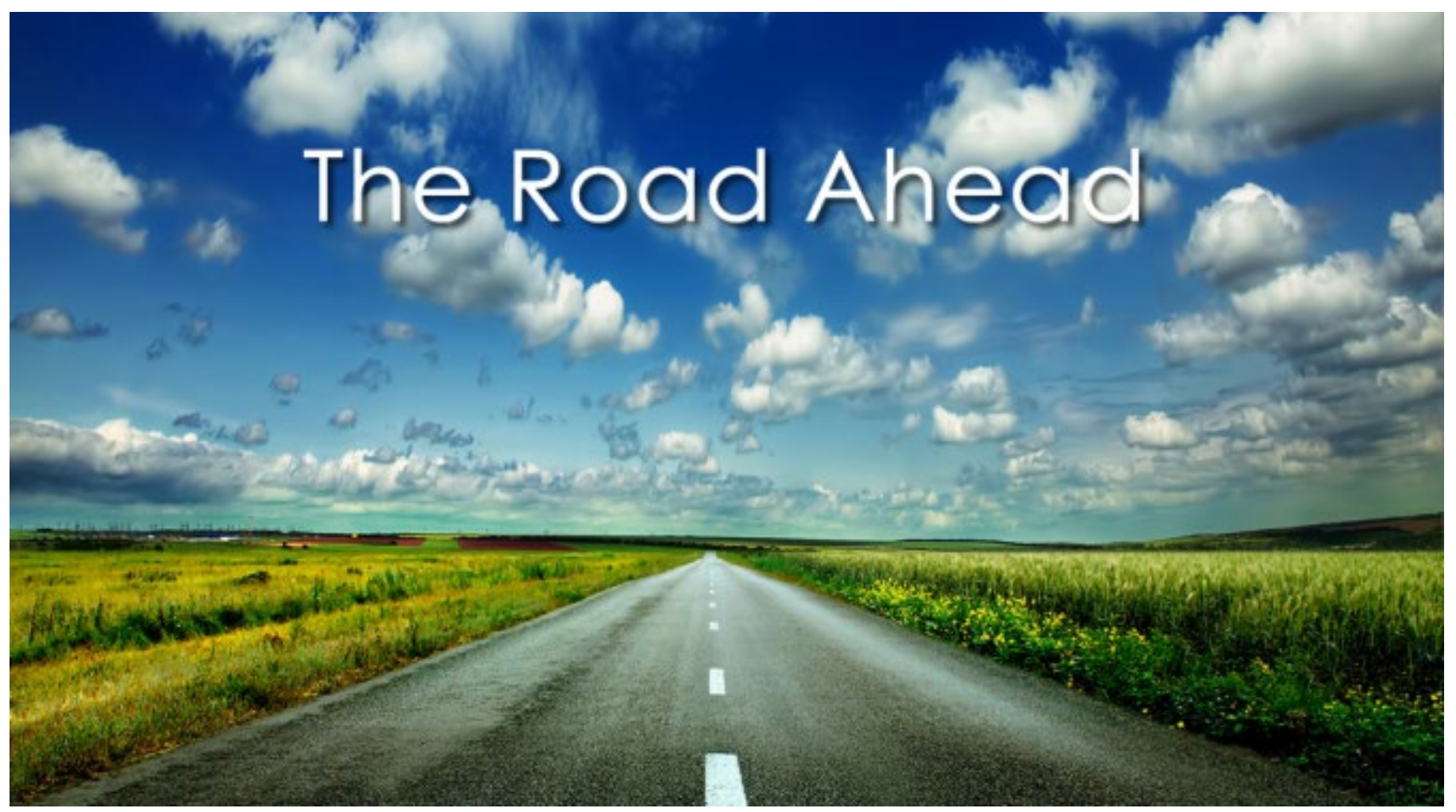

Fermilab has an exciting and promising quantum science program, led by Panagiotis Spentzouris https://qis.fnal.gov/ 


\section{Illinois-Express Quantum Network (IEQNET)}

- Aim to build a metropolitan-scale quantum network testbed that demonstrates important advanced quantum networks capabilities beyond the lab.

- Funded by DOE ASCR, \$3.2M, announced on Sep, 2019.

- Research team

- Fermilab (lead)

- P. Spentzouris (PI), C. Pena, W. Wu

- Caltech

- M. Spiropulu, N. Lauk

- Northwestern University

- P. Kumar, G. Kanter

- Argonne National Lab

- J. Chung, R. Kettimuthu 


\section{IEQNET -- Topology}

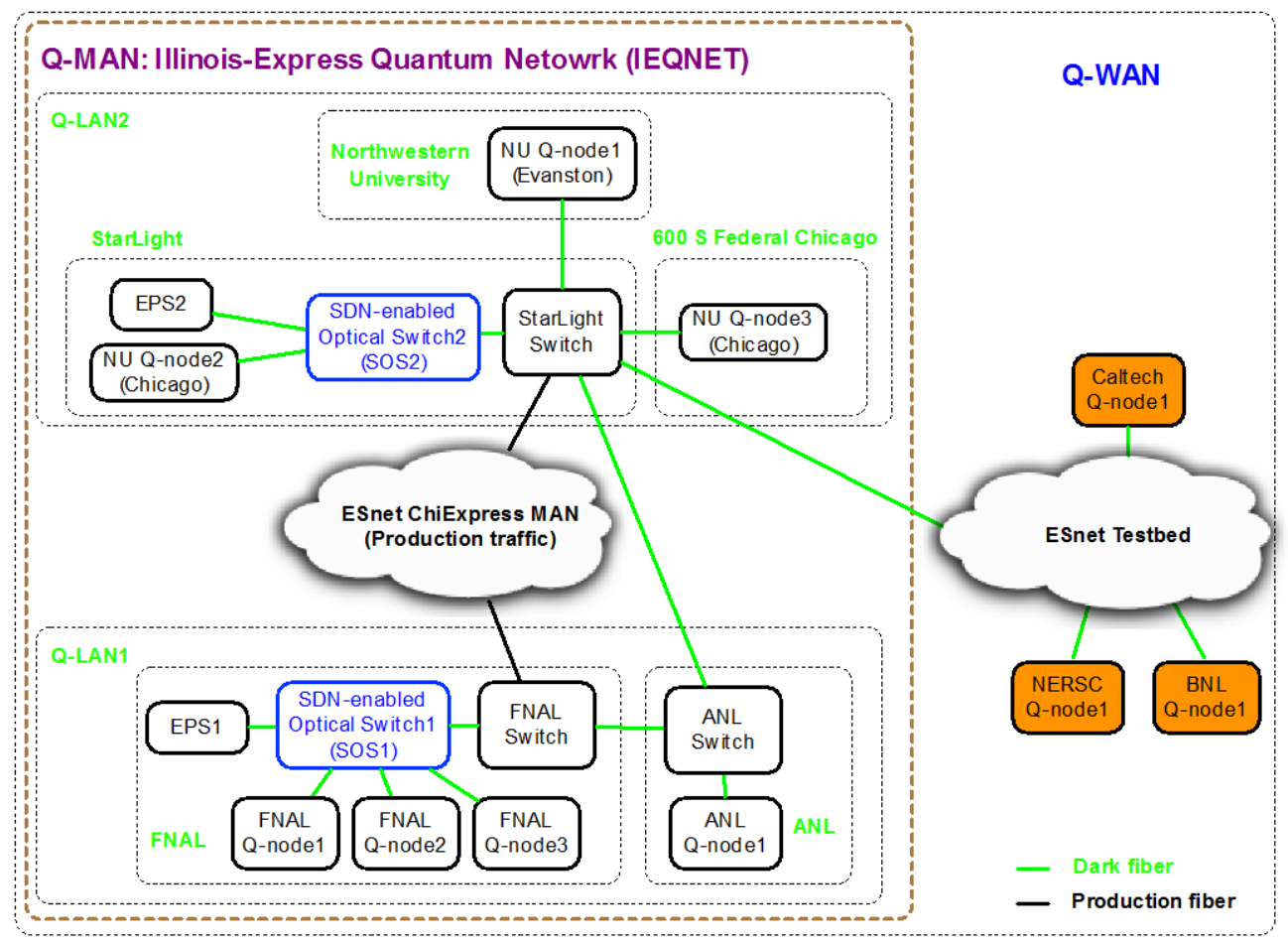

\section{Key features:}

- Support multi-node, flexible, and resilient network configuration

- Support multi-user

- Coexist with traditional networks in the same optical transmission systems

- Adopt a layered architecture and a centralized control 


\section{IEQNET - Model and Architecture}

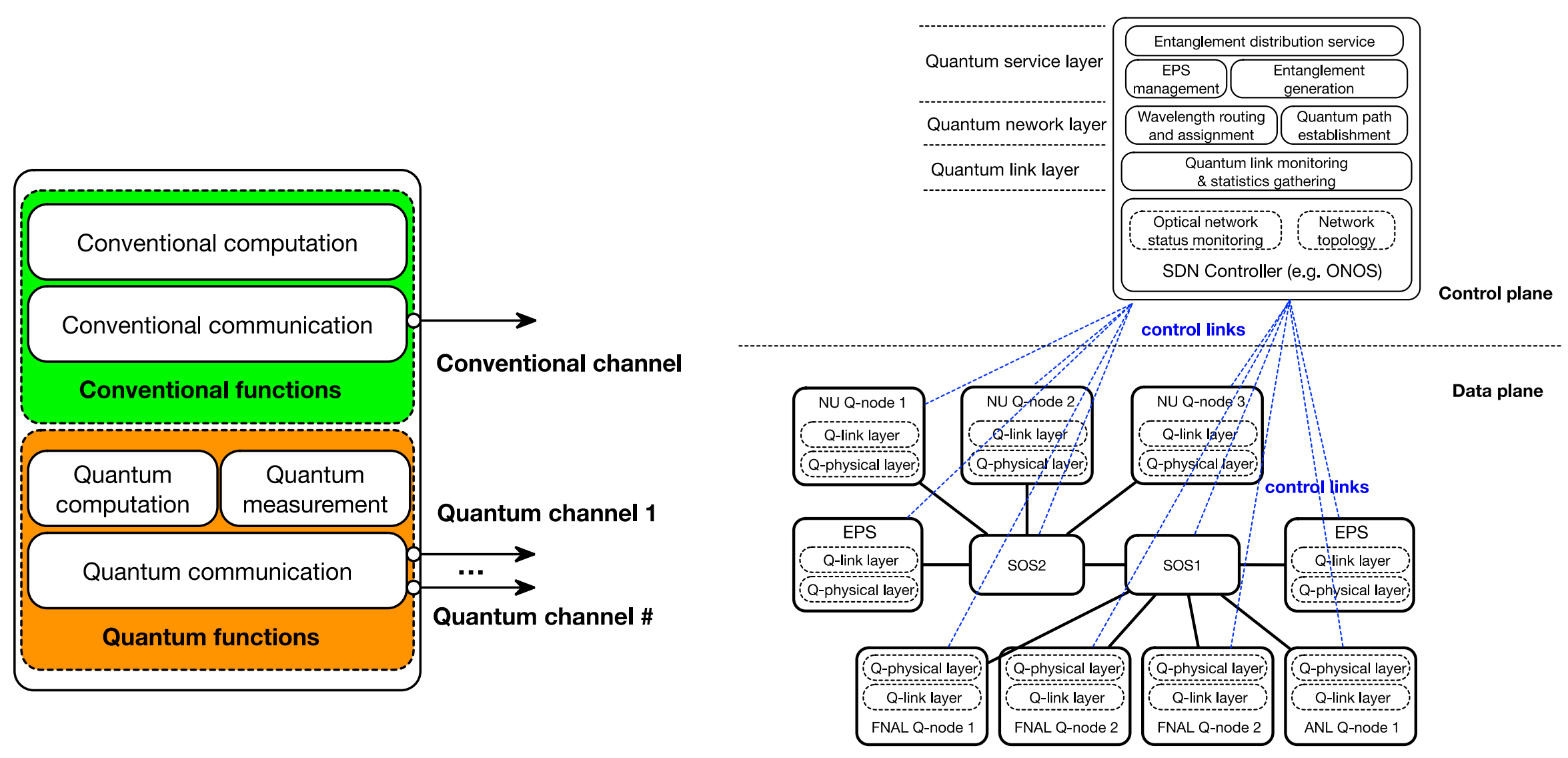

Q-node model

Network Architecture 


\section{IEQNET - Challenges}

- Photon loss

- Increase exponentially with distance travelled

- No-cloning theorem

- Quantum state of photons cannot be amplified without any disturbance

- Phase decoherence

- Degrade or terminate entanglement 


\section{Questions?}

\section{wenji@fnal.gov}

